\title{
El yoga como herramienta para el desarrollo de la conciencia corporal y emocional
}

\section{Yoga as a tool for the development of body and emotional awareness}

\author{
Sara Martínez Huertas ${ }^{1}$ \\ ${ }^{1}$ Universidad International de Valencia.
}

\section{Resumen}

En tiempos de cambio, la educación debe asumir nuevos retos, respondiendo con recursos y metodologías innovadoras. En la sociedad de la información y el conocimiento, las escuelas no solo han de centrarse en trabajar contenidos, sino que han de adoptar una perspectiva global que ayude al alumnado en su proceso de desarrollo. En este contexto, se puede contar con el yoga, un sistema de desarrollo personal milenario que, adaptado a la educación infantil, potencia recursos personales que facilitan el conocimiento de uno mismo y la expresión de las propias emociones. El presente trabajo pretende profundizar en las posibilidades que ofrece el yoga como recurso didáctico innovador para que el alumnado sea más consciente de su cuerpo y de sus emociones. La metodología utilizada es la de investigación-acción participativa. Se ha diseñado y aplicado una intervención de 10 sesiones de yoga que han permitido mejorar la consciencia en la respiración, en el conocimiento de las partes del cuerpo y en la relajación. También se ha comprobado que el recurso del yoga-cuento ha ayudado a mejorar el conocimiento y expresión de las propias emociones, compartiéndolas con los demás. Por último, se ha podido constatar que el yoga genera mayoritariamente emociones agradables y sensaciones de bienestar.

\section{Fecha de recepción:}

09-07-2020

Fecha de aceptación:

25-11-2020

\section{Palabras clave}

Yoga; Educación Infantil;

Conciencia corporal;

Conciencia emocional;

Bienestar.

Keywords

Yoga; Infant Education;

Body awareness; Emotional awareness; Wellness.

\section{Abstract}

In times of change, education has to take on new challenges, responding with new resources and methodologies. Nowadays, in the information and knowledge society, schools have to focus, not only on working content but also have to adopt a global perspective that helps students in their development process. In this context, yoga arises, as a system of personal development that, adapted to early childhood education, enhances personal resources that facilitate self-knowledge and the expression of one's emotions. This work aims to deepen the possibilities offered by yoga as an innovative teaching resource so that students are more aware of their body and their emotions. The methodology used is participatory action research. An intervention of 10 yoga sessions has been designed and applied that have allowed us to improve awareness in breathing, in the knowledge of body parts and in relaxation. It has also been proven that the resource of yoga-story has helped to improve the knowledge and expression of one's emotions, sharing them with others. Finally, it has been found that yoga generates mostly pleasant emotions and feelings of well-being. 


\section{Introducción}

La educación ha de preparar para la vida y para la inserción social, pero nuestras sociedades son cada vez más complejas, diversas y plurales. En la sociedad de la información y el conocimiento todo cambia de forma rápida y las escuelas han de responder a nuevas necesidades, con nuevos recursos y metodologías (López y Moral, 2017).

Delors (1996) define los retos a los que debería responder la educación del siglo XXI, una educación que adopta una perspectiva global para ayudar a la persona en su proceso de desarrollo mediante cuatro dimensiones del aprendizaje que se han de trabajar de forma equilibrada: aprender a conocer, aprender a hacer, aprender a convivir y aprender a ser.

En este nuevo contexto escolar, el yoga puede ayudar a desarrollar la conciencia emocional, en las dimensiones del Conviviry el Ser, y la conciencia corporal, en las dimensiones del Saber y Saber hacer, como un recurso innovador que no solo se centra en la memorización de contenidos sino, sobre todo, en el desarrollo de los recursos personales que se ponen en juego para gestionar las relaciones, con uno mismo y con los demás. El yoga permite desarrollar habilidades intrapersonales, interpersonales y cinestésico-corporales, habilidades identificadas por Gardner (2003) como parte fundamental de las Inteligencias Múltiples.

El yoga aplicado al ámbito escolar tiene cabida en el currículo educativo de la etapa de educación infantil. La orden por la que se establece el currículo y se regula esta etapa (ORDEN ECI 3960/2007) establece tres áreas de aprendizaje: Conocimiento de sí mismo y autonomía personal, Conocimiento del entorno y Lenguajes, comunicación y representación. Estas áreas deben entenderse como ámbitos propios de la experiencia y el desarrollo infantil y del aprendizaje de actitudes, procedimientos y conceptos que contribuirán al desarrollo del alumnado, facilitando su participación activa en el entorno. El área de Conocimiento de sí mismo y autonomía permite trabajar, entre otros, objetivos relacionados con el conocimiento del propio cuerpo y la gestión de emociones. Estos aprendizajes se pueden desarrollar a partir de propuestas basadas en el yoga.

En el presente artículo se pretende profundizar en las posibilidades que ofrece el yoga como recurso didáctico innovador para que el alumnado pueda ser más consciente de su cuerpo y de sus emociones.

\section{Etimología e historia del Yoga}

La palabra yoga, de origen sánscrito, significa "unión" y se refiere a la fusión de las diferentes dimensiones de la persona, que es entendida en toda su globalidad: cuerpo, mente, emociones y espiritualidad (García, 2015). Esta globalidad enlaza con la visión amplia de la 
educación propuesta por Delors, quien pone de manifiesto que las competencias emocionales están implicadas en el desarrollo integral del alumnado.

De forma más concreta, el yoga se puede definir como el conjunto de técnicas aplicadas para conseguir el desarrollo integral de la persona a nivel físico, mental y espiritual y, a la vez, hacer posible una vivencia más consciente (Hernández, 1997).

Esta práctica nació en la India hace más de 5000 años (Hernández, 1997, p.21), pero los primeros textos sobre ella aparecen hacia el 300-400 d. C., cuando Patanjali escribió los Yoga Sutras, que sintetizan el primer método de yoga, estructurado en ocho etapas (Maestre, 2010) que permiten llegar al centro de la persona (Roigé et al., 2018). Estas etapas pueden resumirse de la siguiente forma: 1) Código social para aprender a vivir en armonía con los demás, 2) Código personal para mantener el cuerpo y la mente sanos, 3) Posturas para fortalecer y purificar el cuerpo, 4) Respiración, para equilibrar la energía y las emociones, 5) Relajación, para descansar y recuperar fuerzas, 6) Concentración para fortalecer la mente manteniéndola quieta, 7) Meditación, para experimentar el silencio y 8) Despertar el espíritu.

A finales del siglo XIX, el yoga aparece en el continente europeo por medio de soldados ingleses que habían vivido en la India. Es en el siglo XX, especialmente en los años sesenta, cuando el yoga se expande por el mundo occidental. Con el tiempo, irá perdiendo la carga espiritual inicial, "adoptando una envoltura psicocorporal, adaptable a todo tipo de personas" (Bravo y Pagazaurtundua, 2003, p.35).

La expansión del yoga en Occidente continúa durante el siglo XXI, hasta tal punto que las Naciones Unidas decretan el 21 de junio como el Día Internacional del Yoga, que se considera "un medio sencillo, accesible e inclusivo para fomentar el bienestar y la salud física y espiritual" (Muñoz, 2016, p.118).

Actualmente, se practican diferentes modalidades de yoga en todo el mundo. En el presente trabajo se tomará como punto de partida el yoga psicofísico o Hatha-yoga, una de las modalidades más extendidas en Occidente (Calle, 2014), que desarrolla prácticas como las asanas o posturas, la respiración y la relajación.

\section{Yoga en la escuela}

Las primeras aplicaciones del yoga en ámbito escolar se realizaron en París, en 1973, puestas en marcha por la francesa Micheline Flak, con el fin de mejorar el rendimiento de los alumnos. En 1978 fundó en Francia la RYE (Reserche sur le Yoga dans /'Éducation), asociación que ha generado una metodología práctica a partir de los principios básicos del yoga y se ha expandido a otros países. 
Este método se ha aplicado en las etapas de educación infantil, primaria y secundaria (Muñoz, 2012) con la idea de incidir en los procesos de enseñanza-aprendizaje, potenciando el entusiasmo por aprender y favoreciendo el bienestar y la confianza (Páez y Páez, 2016).

Los referentes pedagógicos de esta metodología se hallan en la adaptación a nuestro presente de los seis primeros pasos del yoga de Patanjali: 1) disciplina externa para vivir juntos, 2) disciplina interna para cuidar el cuerpo y la mente, 3) postura física, 4) conciencia respiratoria, 5) relajación y 6) concentración. Según este método, los dos últimos pasos de Patanjali (meditación y despertar del espíritu) no se aplican en el entorno escolar por tener una carga más espiritual.

Se trata de una propuesta muy amplia, pero no siempre se pueden trabajar todos los pasos, sino que se han de seleccionar y priorizar en función de los objetivos planteados y de las necesidades concretas del grupo, teniendo en cuenta tanto los contenidos curriculares como el estado emocional del alumnado y los docentes (Muñoz, 2012).

Estas aplicaciones del yoga físico en el ámbito escolar han generado determinados efectos positivos. Bravo et al. (2003) clasifican sus beneficios a nivel educativo en tres grandes planos: 1) En el plano físico, permite explorar el cuerpo de forma respetuosa y tomar conciencia de él, 2) En el plano mental, mejoran las habilidades cognitivas a partir del movimiento y 3) En el plano social, beneficia la comunicación y relación respetuosa con los demás.

Por otro lado, García (2011), Endara (2012) y Collado y Cadenas (2013), entre otros, destacan los beneficios que genera en el plano emocional, ya que permite canalizar y expresar emociones generando sensaciones de bienestar.

\section{Método}

\section{Metodología.}

La metodología utilizada es la de investigación-acción participativa, una metodología apropiada para investigaciones a pequeña escala, realizadas en el ámbito educativo. En esta metodología, los investigadores no son externos, sino que participan de la situación objeto de estudio, identificando problemas e iniciando procesos de estudio para su mejora (Bell, 2005).

El diseño metodológico se ha estructurado en tres fases siguiendo la propuesta de Bernal (2010):

- Fase inicial, de contacto con la comunidad y observación. La observación se realiza en el CEIP Congrés-Indians, en la etapa de educación infantil. Durante dos semanas se 
realiza una observación de las sesiones de Movimiento Consciente, en las que se pretende promover el conocimiento a través del cuerpo y los sentidos.

- Fase intermedia, de elaboración del plan. Se plantean los objetivos y se diseña un proyecto de yoga para educación infantil, a partir de las necesidades detectadas. Los objetivos generales y específicos planteados se centran en diseñar y aplicar una intervención que, a partir del yoga, permita desarrollar la conciencia corporal y emocional del alumnado.

- Fase de ejecución y evaluación del estudio. El proyecto se aplica durante 4 meses, con 52 niños del nivel de P4 organizados en 3 grupos de 17 alumnos cada uno. Se elaboran instrumentos de recogida de datos específicos que sirven para valorar la experiencia.

\section{Objetivos.}

A partir de la fase de observación y detección de necesidades se han planteado dos objetivos generales que han guiado el proyecto de investigación:

- Valorar el desarrollo de la conciencia corporal mediante una intervención de 10 sesiones de yoga.

- Diseñar una intervención que permita desarrollar la conciencia emocional del alumnado con la práctica del yoga.

\section{Muestra}

El presente proyecto se aplica en el CEIP Congrés-Indians, un centro educativo público de la ciudad de Barcelona. En concreto, se dirige a los dos grupos de P4, segundo curso del segundo ciclo de educación infantil, de la escuela. Son un total de 51 alumnos de 4 a 5 años, 22 niñas y 29 niños.

En la franja horaria en la que se interviene, estos grupos se mezclan y se dividen en tres grupos de 17 alumnos cada uno. Los grupos han sido elaborados por las maestras, con criterios de heterogeneidad, de forma que queden equilibrados en cuanto a las diferentes características que presentan. Se trata de grupos en los que existe una gran diversidad de ritmos, niveles de desarrollo, habilidades y actitudes. En general, muestran mucha necesidad de movimiento y están aprendiendo a mantener la atención en actividades pautadas.

La maestra referente, acompaña en las sesiones de yoga haciendo una labor de observación, apoyo y atención a los conflictos y necesidades concretas que se presenten. 


\section{Recursos didácticos.}

La aplicación práctica del proyecto se ha llevado a cabo a partir del diseño de 10 sesiones de yoga. En estas sesiones se ha usado una metodología lúdica e integradora, con diferentes recursos, entre los que destacan los siguientes:

- Juego. Se trata del recurso característico de todos los talleres de yoga para niños, que han de partir de la diversión y la alegría (Roigé et al, 2018), para posteriormente poder llegar a la introspección y a la calma.

- Observación e imitación. La imitación en la ejecución de posturas se da en las primeras sesiones, pero, con la repetición, los niños y niñas las aprenden y las hacen sin necesidad de imitar. Además, la maestra sigue ejecutando las posturas como una forma de generar transferencia, que es el proceso por el cual los alumnos y alumnas se identifican con la maestra mediante la práctica y sienten que están pasándolo bien juntos (Calle, 2014).

- Centro de interés. El mundo natural y de los animales se ha convertido en el hilo conductor que ha aportado globalidad a la propuesta y una gran motivación y creatividad por parte del alumnado. Se han usado mayoritariamente posturas de yoga de animales por su efecto motivador y evocador de experiencias y conocimientos previos.

- Técnicas del yoga psicofísico. Se trata del recurso central del proyecto. Se plantean siempre de forma lúdica, adaptadas al nivel y a los intereses del grupo. Se usarán principalmente las técnicas del yoga psicofísico, siguiendo los 5 primeros pasos del método RYE: 1) actitud hacia el exterior, 2) actitud hacia el interior, 3) postura, 4) conciencia respiratoria y 5) relajación.

- Cuento motor. Se trata de un recurso didáctico que convierte al alumnado en protagonista y aprovecha las virtudes pedagógicas del cuento para el desarrollo de las habilidades motrices (Bravo et al., 2003). Este recurso se ha adaptado a partir de los yoga-cuentos, permitiendo realizar posturas de yoga, respiraciones y relajaciones a través de sus diferentes personajes y escenas.

\section{Diseño de las sesiones.}

Los recursos didácticos seleccionados se han introducido en las sesiones a partir de una estructura de tres fases:

1) El ritual de entrada inicial, que sirve para dar la bienvenida, reconocer al grupo y situar el cuerpo y la postura inicial. 
2) Las actividades centrales, que a partir de juegos y/o cuentos permiten llevar a cabo el calentamiento del cuerpo, la realización de posturas de yoga y las técnicas de relajación y respiración.

3) El ritual de salida, que cierra la sesión planteando actividades más relajadas y emocionales como las "charlas del corazón".

A continuación, en la Tabla 1, se especifican las 3 fases de las sesiones y los 6 tipos de actividades específicas que se realizan, indicando su relación con los pasos del RYE.

Tabla 1. Fases, tipos de actividades y relación con los pasos del RYE.

\begin{tabular}{|c|c|c|}
\hline FASES & ACTIVIDADES & PASOS RYE \\
\hline Ritual de entrada & $\begin{array}{l}\text { Postura inicial. Canción de inicio. } \\
\text { Respiración. }\end{array}$ & Actitud hacia el exterior (1) \\
\hline \multirow{4}{*}{$\begin{array}{l}\text { Actividades } \\
\text { centrales }\end{array}$} & $\begin{array}{l}\text { Calentamiento dirigido y lúdico: } \\
\text { Juegos, baile, masajes. }\end{array}$ & Actitud hacia el interior (2) \\
\hline & $\begin{array}{l}\text { Posturas de yoga: Juegos y } \\
\text { cuentos. }\end{array}$ & Postura física y corporal (3) \\
\hline & Respiración: Juegos y retos. & $\begin{array}{l}\text { Conocimiento y control de la } \\
\text { respiración (4) }\end{array}$ \\
\hline & $\begin{array}{l}\text { Relajación: Técnicas, } \\
\text { visualizaciones, respiración. }\end{array}$ & Retracción de los sentidos (5) \\
\hline Ritual de salida & $\begin{array}{l}\text { Cierre: Charlas del corazón } \\
\text { (emociones). }\end{array}$ & Actitud hacia el interior (2) \\
\hline
\end{tabular}

A partir de esta estructura común, se han diseñado y aplicado 10 sesiones que han progresado en complejidad, pasando por tres etapas: una etapa inicial de 2 sesiones de presentación y motivación, seguida de una etapa central de 7 sesiones de yoga-cuentos que han trabajado las emociones y una etapa final, con una última sesión de cierre y evaluación. En la tabla 2, se puede consultar el número y orden de las diferentes sesiones realizadas, con los contenidos transversales y emocionales trabajados. 
Tabla 2. Sesiones numeradas y contenidos

\begin{tabular}{|c|c|c|c|}
\hline & TÍTULO & $\begin{array}{l}\text { CONTENIDO } \\
\text { TRANSVERSAL }\end{array}$ & EMOCIONES \\
\hline 1 & Presentación: ¿Qué es el yoga? & $\begin{array}{l}\text { Animales India. Mi } \\
\text { cuerpo. }\end{array}$ & Sin especificar. \\
\hline 2 & Motivación: Cesto sorpresa. & Animales & Sin especificar. \\
\hline 3 & Yoga-cuento: Pequeño búho. & Bosques de Europa. & Miedo, tristeza, alegría. \\
\hline 4 & Yoga-cuento: El león sin miedo. & Sabana africana. & Miedo y valor. \\
\hline 5 & Yoga-cuento: La rana Tiddalik. & Mares de Oceanía. & Enfado y alegría. \\
\hline 6 & Yoga-cuento: Viaje de mariposa. & Ecosistemas de América. & Enfado, miedo, tristeza y alegría. \\
\hline 7 & Yoga-cuento: Señor viento. & $\begin{array}{l}\text { Fenómenos } \\
\text { atmosféricos. }\end{array}$ & Enfado y autoestima. \\
\hline 8 & Yoga-cuento: El tigre sin rayas. & Selvas de Asia. & $\begin{array}{l}\text { Tristeza, superación } \\
\text { autoconcepto. }\end{array}$ \\
\hline 9 & Yoga-cuento: Manzana Viajera. & El ciclo de la vida. & Miedo, valentía y curiosidad. \\
\hline 10 & Cierre y evaluación. & Mi cuerpo. & Sin especificar. \\
\hline
\end{tabular}

Se plantean sesiones muy dinámicas en las que se realizan actividades cortas y variadas para mantener la atención del alumnado. Por un lado, en todas las sesiones se da la repetición de la misma estructura, que aporta seguridad, ya que, al realizar siempre las mismas fases, el alumnado puede anticipar. Por otro lado, se juega con la sorpresa, ofreciendo propuestas y materiales diferentes e incorporando pequeños elementos nuevos en cada sesión, para aumentar la motivación. En todo caso, las sesiones siguen un hilo conductor temático, a través de los contenidos transversales, que aporta sentido y globalidad a las sesiones.

\section{Instrumentos de recogida de datos.}

La recogida de información se realiza a través de la observación directa y participativa. Para registrar dicha información se usarán los siguientes instrumentos:

- Diario de campo de las sesiones. Se trata de un instrumento de registro cualitativo ad hoc, un documento en el que se recogen las principales informaciones de las sesiones 
una vez realizadas: número de participantes, duración, desarrollo de la sesión, sensaciones generales, registro de la actividad de clima emocional y efectos fuera de la sesión.

- Cuadro de registro de observaciones. Permite recoger información específica sobre los comportamientos mostrados por los niños y niñas durante las tres últimas sesiones. La observación es realizada por la maestra de apoyo. Los aspectos que se observan, valoran y registran son: expresión corporal, conciencia corporal, realización de posturas de yoga y conciencia emocional. Dos de estos aspectos se registran de forma cuantitativa, marcando un número (1, 2, 3 o más de 3): número de partes del cuerpo que moviliza en el calentamiento y número de posturas de yoga realizadas a lo largo de la sesión. El resto de ítems se registra de forma cualitativa, valorando la consecución del objetivo en tres niveles (no alcanzado, en proceso y alcanzado): reconoce las propias emociones y se expresa con el cuerpo a través de gestos y movimientos.

- Cuadros de registro de la actividad de clima emocional. Recogen información relacionada con las emociones y sensaciones del alumnado al acabar las sesiones de los yoga-cuentos. Mediante la dinámica de las "charlas del corazón", el alumnado hace un esfuerzo de conciencia emocional y escoge la imagen de un paisaje, acompañada de palabras, para situarse en un bloque de sensaciones corporales y emocionales. De esta manera, identifica y expresa cómo se siente en ese momento. Se ofrecen cuatro bloques de sensaciones y emociones: 1) Tranquilo, calmado o relajado, a través de la imagen del lago en calma; 2) Alegre, con energía o animado, a través de la imagen de campo con flores y sol, 3) Triste, enfadado o cansado, a través de la imagen de flor marchita; 4) Nervioso, tenso o alterado, a través de la imagen de rayos en el cielo negro.

- Cuadro de análisis de los dibujos sobre su cuerpo. En la primera y última sesión se pide al alumnado que dibuje su cuerpo. Las maestras escriben las partes del cuerpo que cada uno de ellos verbaliza al acabar el dibujo. De esta forma, se dispone de una evaluación diagnóstica inicial (primera sesión) y una evaluación final (última sesión) respecto a la consciencia corporal. En el cuadro de análisis se registra la información de cada dibujo, indicando las partes del cuerpo que se identifican gráficamente y las palabras verbalizadas. Entre paréntesis se indica el número de partes identificadas, gráfica y verbalmente.

\section{Resultados}

Las 10 sesiones de yoga diseñadas se han aplicado en tres grupos, de forma que se han realizado un total de 30 intervenciones. Durante todo el proceso se han utilizado los 
instrumentos de recogida de información previamente diseñados, de manera que se dispone de datos tanto cualitativos como cuantitativos que han evidenciado los siguientes resultados. Los datos recogidos en el diario de campo nos demuestran que a partir de estas sesiones se han producido avances en la conciencia corporal y emocional. Respecto a la conciencia corporal, el alumnado ha movilizado diferentes partes del cuerpo de forma más consciente durante las actividades de calentamiento y preparación del cuerpo y durante la realización de las posturas de yoga. También se ha observado un avance en la exploración del propio movimiento y en las posibilidades que ofrece el lenguaje corporal. Igualmente, el alumnado ha avanzado respecto a la conciencia respiratoria, conociendo y vivenciando los movimientos que genera la respiración en el cuerpo, y ha mostrado comportamientos cada vez más autónomos en la realización de las técnicas de relajación.

Los cuadros de observación han permitido registrar determinados comportamientos en las tres últimas sesiones y permiten constatar que:

- El 85\% del alumnado se expresa mediante su cuerpo, siendo, de esta manera, más consciente del lenguaje corporal y, por lo tanto, de su cuerpo y de las posibilidades expresivas y comunicativas que ofrece.

- Respecto a la conciencia corporal y al conocimiento de las diferentes partes del cuerpo, el 60\% del alumnado reconoce y mueve más de 3 partes del cuerpo en las dinámicas de calentamiento y preparación del cuerpo (Figura 1).

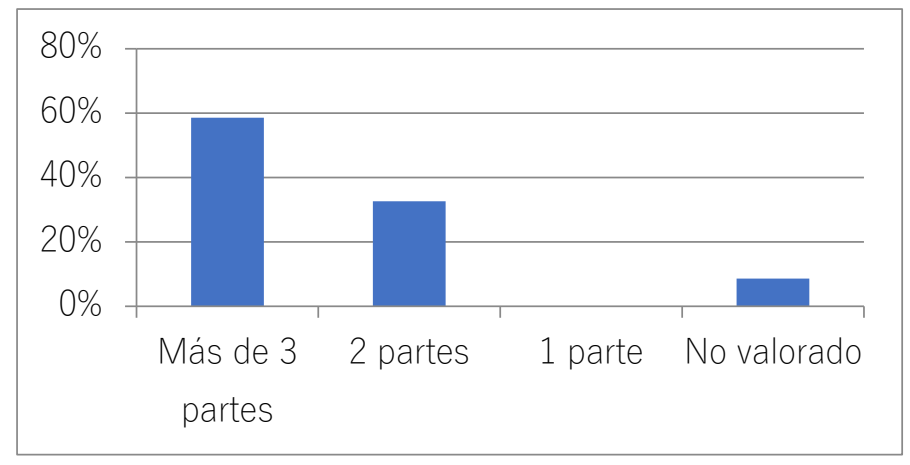

Figura 1. Número de partes del cuerpo que reconoce y moviliza.

- El 84,70\% del alumnado realiza más de 3 posturas de yoga por sesión (Figura 2). Cabe señalar que al realizar posturas de yoga se movilizan diferentes partes del cuerpo de forma consciente y coordinada. 


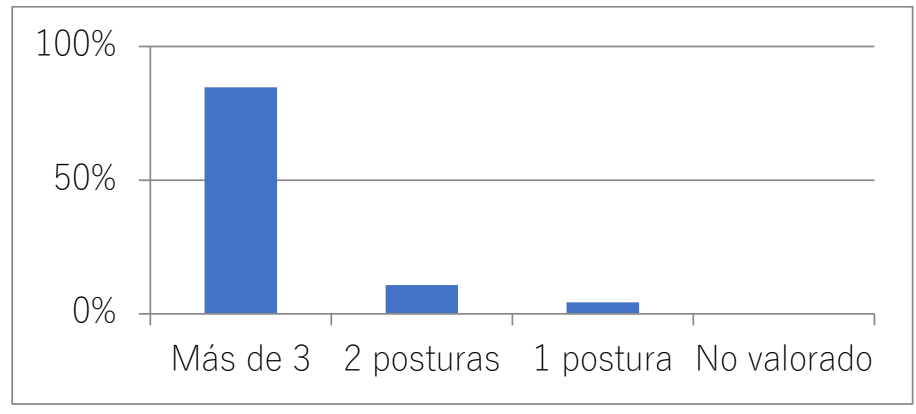

Figura 2. Número de posturas de yoga que realiza.

- El 97,80\% del alumnado reconoce y expresa sus emociones de forma clara al finalizar las sesiones de los yoga-cuento, mediante la dinámica de "las charlas del corazón".

Los cuadros de análisis de los dibujos sobre su cuerpo permiten constatar una mejora sustancial en la conciencia corporal: en la primera sesión la mayoría de los niños identifican menos de 4 partes del cuerpo, mientras que en la última sesión la mayoría identifica más de 7 partes del cuerpo, siendo muchos los que identifican más de 10 partes de su cuerpo (tabla 3). En la última sesión se ha constatado una evolución hacia un nivel de conocimiento muy alto respecto a las partes de su propio cuerpo.

Tabla 3. Número de niños que reconocen diferentes partes de su cuerpo.

\begin{tabular}{lcccccc}
\hline TOTAL & $\begin{array}{c}\text { Ninguna } \\
\text { parte }\end{array}$ & $\begin{array}{c}\text { De 1 a 3 } \\
\text { partes }\end{array}$ & $\begin{array}{c}\text { De 4 a 6 } \\
\text { partes }\end{array}$ & $\begin{array}{c}\text { De 7 a 9 } \\
\text { partes }\end{array}$ & $\begin{array}{c}10 \text { o más } \\
\text { partes }\end{array}$ \\
\cline { 2 - 6 } $\begin{array}{l}\text { Nivel de } \\
\text { conocimiento }\end{array}$ & MUY BAJO & BAJO & MEDIO & ALTO & MUY ALTO \\
$\begin{array}{l}\text { Sesión inicial (44 } \\
\text { alumnos) }\end{array}$ & 15 & 10 & 10 & 7 & 2 \\
$\begin{array}{l}\text { Sesión final (45 } \\
\text { alumnos) }\end{array}$ & 5 & 3 & 7 & 13 & 17 \\
\hline
\end{tabular}

Por último, los cuadros de registro del clima emocional han permitido recoger las respuestas del alumnado respecto a las sensaciones y emociones que sentían al finalizar las sesiones de los yoga-cuentos. En el siguiente gráfico (Figura 3) se muestran los bloques de emociones y sensaciones escogidos por el alumnado, ordenados de mayor a menor respuesta: 


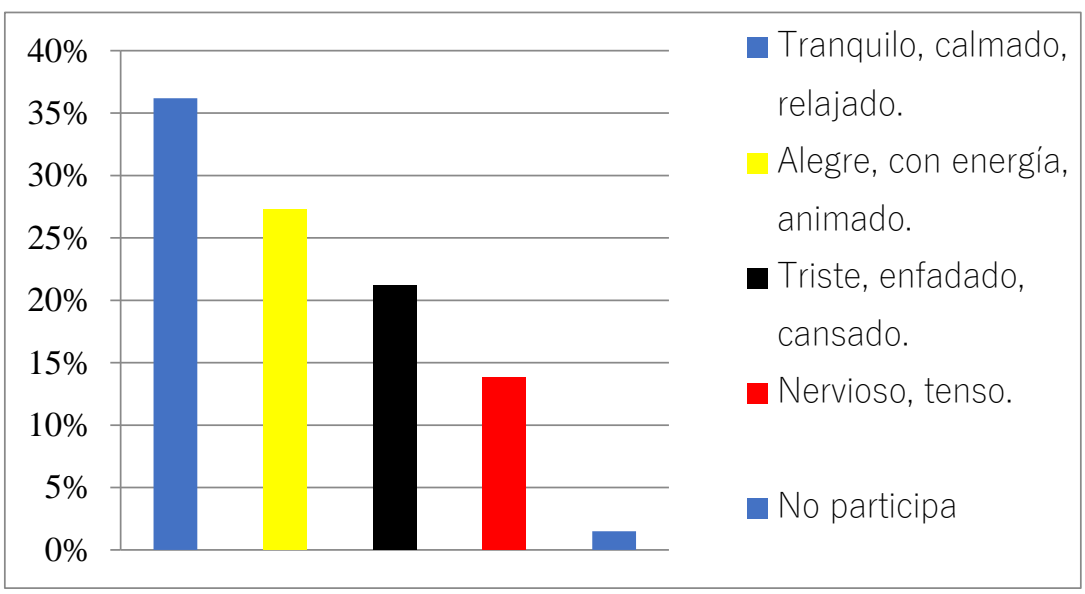

Figura 3. Emociones y sensaciones al finalizar las sesiones de yoga-cuentos.

Se puede afirmar que el 63,5\% de las respuestas del alumnado al finalizar estas sesiones se relacionan con emociones agradables: en primer lugar, la calma (36,2\%) y en segundo lugar la alegría (27,3\%).

En relación con el desarrollo de la conciencia emocional, los registros cualitativos del diario de campo aportan observaciones y matices sobre el papel que ejerce la práctica del yoga en este sentido. De hecho, se ha observado que mediante las sesiones realizadas no solo se ha mejorado la conciencia emocional, sino también la expresión emocional, siendo dos conceptos íntimamente relacionados. A partir de la actividad de "las charlas del corazón" el alumnado ha podido identificar y reconocer sus emociones en ese momento. Además, se ha hablado de sus causas y de diferentes formas de gestionarlas.

Durante las sesiones, se han dado muchos ejemplos: se ha hablado de la tristeza y se ha visto que está relacionada con la pérdida ("no está mi mamá"), se ha visto que el enfado se produce cuando hay un daño ("un amigo me ha pegado") y también se ha comprobado que la alegría se produce cuando hay una satisfacción ("estoy alegre porque tengo música"). Al tener más consciencia de las propias emociones y de sus causas, es más fácil buscar alternativas que ayuden a gestionarlas mejor. A partir de las emociones que expresaba el alumnado, las maestras daban ideas para gestionarlas de forma más adaptativa. Por ejemplo: "Si estás nervioso, puedes ir al exterior", "Si estás cansado, puedes descansar en un espacio tranquilo". Todo este conocimiento ayuda a que el alumnado sea más consciente de las propias emociones y sensaciones y, así, conviva mejor con ellas. 


\section{Discusión}

Los resultados obtenidos son coherentes con otras experiencias e investigaciones publicadas sobre el yoga.

Tanto los registros del diario de campo y de los cuadros de observación como los análisis de los dibujos realizados sobre su cuerpo nos muestran un desarrollo en el conocimiento de su propio cuerpo, tanto a nivel cognitivo y verbal (reconocer y nombrar diferentes partes del cuerpo) como a nivel corporal y expresivo (movilizar las diferentes partes, de forma aislada y de forma coordinada, y aportar un sentido comunicativo). Estos resultados son coherentes con la visión que nos ofrecen diversos autores que, desde diferentes ámbitos, afirman que la práctica del yoga desarrolla una mayor conciencia corporal (Calle, 2004; García, 2011; Pallasá y Tolosana, 2017; Roigé et al., 2018).

Igualmente, el análisis de los dibujos realizados por los niños y niñas en la primera y última sesión ha demostrado un aumento del conocimiento de las partes del cuerpo, manifestadas de forma gráfica. Estos resultados, permiten afirmar que el alumnado, a lo largo de las sesiones, ha desarrollado el concepto de esquema corporal, entendido como "la representación mental del propio cuerpo, de sus segmentos, de sus posibilidades de movimiento y de sus limitaciones espaciales" (Llorens y Collado, 2016, p.31). Al realizar los dibujos, los niños y las niñas muestran las representaciones mentales que tienen de su cuerpo y de sus segmentos.

Por otro lado, evidencias aportadas por los diferentes instrumentos de recogida de datos llevan a afirmar que las sesiones diseñadas han permitido mejorar la conciencia emocional del alumnado. Este resultado se relaciona con algunos de los resultados obtenido por White (2012), en un estudio realizado en escuelas de secundaria de Estados Unidos. White concluye que con la práctica del yoga se mejora la conciencia de las emociones difíciles. En este caso, a partir del proyecto realizado, se puede afirmar que el yoga no solo ha mejorado la consciencia de las emociones difíciles, sino que ha mejorado el conocimiento y la conciencia de todas las emociones.

Por último, también se ha demostrado que el recurso del yoga-cuento permite generar, de forma mayoritaria, sensaciones de bienestar y emociones agradables como la alegría, la relajación y la calma (63,5\% de las respuestas). Estos resultados son coherentes con los resultados que expone Nieto (2012), que al aplicar un proyecto de yoga en una escuela de Ecuador, comprueba que los estudiantes experimentan mayoritariamente una sensación de bienestar y relajación. 


\section{Conclusiones}

Las evidencias muestran que este proyecto de yoga aplicado al ámbito escolar ha servido para mejorar la conciencia corporal y emocional del alumnado.

Respecto a la conciencia corporal, el alumnado ha aumentado las partes del cuerpo que reconoce y moviliza, ha mejorado la conciencia en la respiración y ha ganado autonomía en la aplicación de las técnicas de relajación.

Respecto a la conciencia emocional, se ha comprobado que el recurso del yoga-cuento ha ayudado al alumnado a identificar y expresar sus emociones, siendo más consciente de las mismas. Además, se ha comprobado que esta propuesta ha generado emociones mayoritariamente agradables (calma y alegría). Este es un resultado coherente con el de Endara (2012) y de vital importancia en sociedades en las que los niños y niñas se ven sometidos a altos niveles de estrés.

De esta manera, el yoga se manifiesta como una herramienta útil para que el alumnado conozca mejor y use de forma práctica recursos mediante los cuales puede generar sensaciones de bienestar y relajación. Estas habilidades son fundamentales en nuestras sociedades, sometidas a altos niveles de estrés y a cambios continuos y acelerados. Este contexto implica efectos en las vidas de los niños y niñas que, en muchas ocasiones, presentan altos niveles de cansancio y tensión.

Ayudar a alcanzar sensaciones de bienestar es fundamental para que los niños y niñas puedan crecer y desarrollarse de forma sana y harmónica, aprendiendo recursos que les ayuden a cuidar de su salud, entendida como un estado de bienestar físico, psíquico y social.

En este sentido, el yoga aplicado en la escuela permite mejorar la conciencia corporal y emocional y, a partir de ese conocimiento, desarrollar en el alumnado recursos prácticos que mejoren su bienestar y su salud. Estos resultados adquieren importancia en estos días de incertidumbre en los que todos, niños y adultos, necesitamos recursos para sentirnos bien.

\section{Agradecimientos}

Este artículo es fruto de mi trabajo de fin de grado (TFG) y quiero agradecer la inestimable ayuda y el acompañamiento de mi directora, Laura Ruiz Sanchís. También quiero manifestar mi profundo agradecimiento al CEIP Congrés-Indians por la oportunidad de aplicar este proyecto. 


\section{Conflicto de interés}

Los autores declaran no tener conflicto de interés.

\section{Financiación}

Este trabajo no ha recibido ningún tipo de financiación.

\section{Referencias}

Bell, J. (2005). Cómo hacer tu primer trabajo de investigación. (Roc Filella Escolá, trad.). México: Gedisa.

Bernal, C. (2010). Metodología de la investigación. Colombia: Pearson Educación.

Bravo, E. y Pagazaurtundua, V. (2003). El cuento motor y el yoga en la enseñanza primaria. Apunts, 2(72), 35-40.

Calle, R. A. (2014). Yoga para niños. Barcelona: Editorial Kairós.

Collado, D. y Cadenas, C. (2013). Educación de las emociones ¿Un reto? E-motion. Revista de Educación, Motricidad e Investigación, 1, 196-211. https://doi.org/10.33776/remo.v0i1.2259

Delors, J. (1996.). La educación encierra un tesoro. Informe a la UNESCO de la Comisión Internacional sobre la Educación para el siglo XXI. Madrid: Santillana/UNESCO.

Endara, M. (2012). Yoga en el aula. (Tesis doctoral, Universidad San Francisco de Quito, Ecuador).

Recuperado

de http://repositorio.usfq.edu.ec/bitstream/23000/1485/1/103712.pdf

García, D. (2011). loga i educació. Un aprenentatge per a la vida. Barcelona: Publicacions de l' Abadia de Montserrat.

García, D. (2015). loga i educació. Un aprenentatge per a la vida. Comunicació Educativa, 28, 47-52. https://doi.org/10.17345/comeduc201547-52

Gardner. Gardner, H. (2003). La inteligencia reformulada. Las inteligencias múltiples en el siglo XXI. Barcelona: Paidós.

Hernández, D. (1997). Claves del yoga. Teoría y práctica. Barcelona: La liebre de marzo. Maestre, J. (2010). Yoga para niños. Málaga: Ediciones Aljibe.

Muñoz, A. (2016). Mitos, hitos y gritos en la internacionalización del yoga. 'llu. Revista de Ciencias de las Religiones, 21, 115-128. https://doi.org/10.5209/ILUR.53848

Muñoz, E. G. (2012). Yoga en el aula. Cuadernos de Pedagogía, 419, 40-43.

ORDEN ECI/3960/2007, de 19 de diciembre, por la que se establece el currículo y se regula la ordenación de la educación infantil. Boletín Oficial del Estado. Madrid, 5 de enero de 2008, núm. 5, pp. 1016-1036. 
Páez, N. S. y Páez, R. M. (2016). Yoga para niños: mediación que minimiza la violencia escolar. Educación y ciudad, 31, 153-164.

Pallasá, M. y Tolosana, J. (2017). Yoga en la escuela. Aproximación desde el proyecto de salud de centro. Aula de Innovación Educativa, 259, 44-47.

Roigé, M. y Civil, A. (2018). Taller de yoga para niños. Barcelona: Alba Balleres.

White, L.S. (2012). Reducing stress in school-age girls through mindful yoga. Journal of Pediatric Health Care, 26(1), 45-56. https://doi.org/10.1016/j.pedhc.2011.01.002 